OPEN ACCESS

Edited by:

Raffi Gugasyan,

Burnet Institute, Australia

Reviewed by:

Ju Qiu,

Shanghai Institutes for Biological

Sciences (CAS), China

Xing Chang,

Shanghai Institutes for Biological

Sciences (CAS), China

*Correspondence:

Liangjing Lu

Iu_liangjing@163.com;

Xiaoxiang Chen

xiaoxiang0721@126.com

Di Yu

di.yu@anu.edu.au

tThese authors have contributed equally to this work.

Specialty section: This article was submitted

to T Cell Biology,

a section of the journal

Frontiers in Immunology

Received: 25 January 2018 Accepted: 16 May 2018

Published: 04 June 2018

Citation:

Deng J, Fan C, Gao X, Zeng $Q$, Guo R, Wei Y, Chen Z, Chen Y,

Gong D, Feng J, Xia Y, Xiang S, Gong S, Yuan L, Shen W, Shen W, Lin L, Jiang T, He D, Lu L, Chen X and Yu D (2018) Signal Transducer

and Activator of Transcription 3

Hyperactivation Associates

With Follicular Helper

$T$ Cell Differentiation and Disease Activity in Rheumatoid Arthritis.

Front. Immunol. 9:1226. doi: 10.3389/fimmu.2018.01226

\section{Signal Transducer and Activator of Transcription 3 Hyperactivation Associates With Follicular Helper T Cell Differentiation and Disease Activity in Rheumatoid Arthritis}

\author{
Jun Deng ${ }^{1,2,3+}$, Chaofan Fan ${ }^{2 t}$, Xin Gao ${ }^{1,2,4 t}$, Qunxiong Zeng ${ }^{1,2}$, Ruru Guo ${ }^{2}$, Yunbo Wei ${ }^{5}$, \\ Zhian Chen ${ }^{1,4}$, Yanan Chen ${ }^{2}$, Dongcheng Gong ${ }^{1,2}$, Jia Feng ${ }^{6}$, Yan Xia ${ }^{6}$, Shifei Xiang ${ }^{6}$, \\ Shushi Gong ${ }^{6}$, Lin Yuan ${ }^{3}$, Wei Shen ${ }^{7}$, Wenyan Shen ${ }^{7}$ Lin Lin ${ }^{8}$, Ting Jiang ${ }^{9}$, Dongyi He ${ }^{9}$, \\ Liangjing Lu ${ }^{2 *}$, Xiaoxiang Chen ${ }^{2 *}$ and $D i Y_{u^{1,2,3,4,5 *}}$
}

\begin{abstract}
'China-Australia Centre for Personalised Immunology, Renji Hospital, Shanghai Jiao Tong University School of Medicine, Shanghai, China, ${ }^{2}$ Department of Rheumatology, Shanghai Institute of Rheumatology, Renii Hospital, Shanghai Jiao Tong University School of Medicine, Shanghai, China, ${ }^{3}$ Hubei Provincial Key Laboratory of Occurrence and Intervention of Rheumatic Diseases, Affiliated Hospital of Hubei University for Nationalities, Enshi, China, ${ }^{4}$ Department of Immunology and Infectious Disease, John Curtin School of Medical Research, The Australian National University, Canberra, ACT, Australia, ${ }^{5}$ Laboratory of Immunology for Environment and Health, Shandong Analysis and Test Center, Qilu University of Technology, Shandong Academy of Sciences, Jinan, China, ${ }^{6}$ Department of Rheumatology, Affiliated Hospital of Hubei University for Nationalities, Enshi, China, ${ }^{7}$ Department of Laboratory Medicine, Renji Hospital, Shanghai Jiao Tong University School of Medicine, Shanghai, China, ${ }^{8}$ Department of Laboratory Medicine, Ruijin Hospital, Shanghai Jiao Tong University School of Medicine, Shanghai, China, ${ }^{9}$ Guanghua Hospital of Integrative Chinese and Western Medicine, Shanghai, China
\end{abstract}

Follicular helper T (Tfh) cells are the specialized CD4+ $T$ cell subset that supports B cells to produce high-affinity antibodies and generate humoral memory. Not only is the function of Tfh cells instrumental to mount protect antibodies but also to support autoantibody production and promote systemic inflammation in autoimmune diseases. However, it remains unclear how the activation of Tfh cells is driven in autoimmune diseases. Here, we report that in patients with rheumatoid arthritis (RA), excessive generation of CXCR5+PD-1+ memory Tfh cells was observed and the frequency of memory Tfh cells correlated with disease activity score calculator for RA (DAS28). The differentiation of Tfh cells is dependent on signal transducer and activator of transcription 3 (STAT3), the key transcription factor downstream of cytokine signal pathways. A drastic increase of phosphorylated STAT3 (pSTAT3) in CD4 ${ }^{+}$T cells were detected in RA patients who also produced larger amounts of STAT3-stimulating cytokines, including IL-6, IL-21, IL-10, and leptin than those of healthy controls. Importantly, the phosphorylation status of STAT3 in CD4 ${ }^{+}$T cells positively correlated with the plasma concentration of IL- 6 and the frequency of memory Tfh cells. This study reveals an IL-6-pSTAT3-Tfh immunoregulatory axis in the pathogenesis of RA and reinforces its candidature as biomarkers and targets for diagnosis and therapy.

Keywords: rheumatoid arthritis, patient, follicular helper T cells, signal transducer and activator of transcription 3 , phosphorylation, IL-6 


\section{INTRODUCTION}

Rheumatoid arthritis (RA) is a chronic and immune-mediated arthritis that affects up to $1 \%$ of the population. It is characterized by synovial inflammation and hyperplasia as well as cartilage and bone destruction (1). Although the name "arthritis" speaks for itself that local tissue remodeling and damage represent the major pathology of RA, the systemic activation of the immune system is instrumental for the disease pathogenesis (1-3). Prior to the onset of clinical disease, increased generation of effector $\mathrm{CD}^{+}$ $\mathrm{T}$ cells, elevated production of pro-inflammatory cytokines, and the presence of anticitrullinated protein antibodies (ACPAs) can usually be detected in blood, therefore, being termed as the "prerheumatoid arthritis" phase (4).

Follicular helper $\mathrm{T}$ (Tfh) cells are a functional $\mathrm{CD}^{+} \mathrm{T}$ cell subset that is specialized to support B cells to generate longlived plasma cells and memory $B$ cells and to produce highaffinity antibodies. By upregulating the chemokine receptor CXCR5, Tfh cells selectively migrate into B-cell follicles and help B cells through the expression of costimulatory receptors including CD40L and OX40 and the secretion of cytokines such as IL-21 (5). The critical role of excessive Tfh cells in the induction of systemic inflammation and the development of autoimmune diseases has been proven by many mouse models (5-8). For RA, there is also accumulated evidence to support a significant contribution of Tfh cells to the development of RA. First, the dependence of Tfh cells has been extensively studied in mouse models. In the commonly used collagen-induced arthritis model, upon the immunization of collagen, $\mathrm{CD}^{+} \mathrm{T}$ cells differentiate into Tfh cells to initiate the pathogenic anti-collagen antibody responses $(9,10)$. In the $\mathrm{K} / \mathrm{BxN}$ autoimmune arthritis model [KRN T cell receptor transgenic mice on the $\mathrm{C} 57 \mathrm{BL} / 6$ (B6) background $\times$ nonobese diabetic mice], KRN $\mathrm{T}$ cells recognize self-antigen glucose-6-phosphate isomerase (GPI) and differentiate into Tfh cells to promote B cells to produce anti-GPI autoantibodies (11-14). Importantly, blocking of Tfh cell generation was able to prevent the development of diseases in these mouse models. Second, there is a strong involvement of B cells in human RA pathogenesis. The production of high-affinity IgG and $\operatorname{IgA}$ antibodies against citrullinated, carbamylated, and acetylated proteins is likely also dependent on Tfh cells (2). Third, several studies including ours have reported excessive Tfh differentiation and function in RA patients as compared to healthy controls (15-19). The association between increased circulating Tfh cells and the presence of high-titer ACPAs and disease activity again suggested a pathogenic role of Tfh cells in RA. The pressing question that remains to be unanswered is the causation of the aberrant Tfh activation in RA.

The development of Tfh cells is driven by signallings via $\mathrm{T}$ cell receptors through sustained antigen stimulation and co-receptors including CD28 and inducible T-cell costimulator (ICOS). The cytokine milieu also shapes the Tfh differentiation. IL-6 and IL-21 induce the activation of signal transducer and activator of transcription 3 (STAT3) and promote the differentiation of mouse Tfh cells (5). For human Tfh cells, additional cytokines including STAT4-stimulating IL-12 and
SMAD2/3-stimulating TGF $\beta$ and Activin A were reported to also contribute $(5,20,21)$. The activation of STAT3 is essential as the capability of Tfh differentiation was greatly impaired in STAT3-deficient mice (22) and patients carrying functional STAT3 deficiency (23). STAT3 has a strong implication in autoimmune diseases. Monogenic activating STAT3 mutations were identified in individuals with a spectrum of early-onset autoimmune disease including juvenile-onset arthritis (24). The involvement of the activation of STAT3 and RA was also supported by studies showing enhanced expression of phosphorylated STAT3 (pSTAT3) and STAT3-inducible gene signature in RA patients (25-28). We, therefore, speculated that the hyperactivation of the STAT3 signaling may lead to abnormal Tfh differentiation in RA patients.

In this study, we examined and confirmed the excessive $\mathrm{Tfh}$ function in RA patients, shown by the increased frequency of circulating memory Tfh cells and its correlations with disease activity. We found a drastic enhancement of STAT3 phosphorylation in $\mathrm{CD}^{+} \mathrm{T}$ cells in RA patients and the activation status of STAT3 positively correlated with the generation of Tfh cells. Major STAT3-stimulating cytokines including IL-6, IL-21, IL-10, and leptin were increased in RA patients and thus contributed to the STAT3 hyperactivation.

\section{RESULTS}

\section{Characteristics of the Study Subjects}

The demographic characteristics of healthy individual controls and patients with RA are shown in Table 1. Thirty-one RA patients including 25 females and 6 males participated the study. Their median age was 60 years. The disease activities

TABLE 1 | Demographics and clinical data of the study cohorts.

\begin{tabular}{|c|c|}
\hline Characteristic & Value \\
\hline \multicolumn{2}{|l|}{ Healthy controls } \\
\hline Female/male, $n$ & $22 / 8$ \\
\hline Age, median (range) & 55 (18-73) \\
\hline \multicolumn{2}{|l|}{ Patients with rheumatoid arthritis } \\
\hline Female/male, $n$ & $25 / 6$ \\
\hline Age, median (range) & $60(22-83)$ \\
\hline Disease duration (year), median (range) & $1(0-10)$ \\
\hline $\mathrm{RF}, n(\%)$ & $25(81 \%)$ \\
\hline Anti-CCP, $n(\%)$ & $26(84 \%)$ \\
\hline DAS28, median (range) & $4.92(1.27-7.88)$ \\
\hline Remission (<2.6), $n(\%)$ & $4(13 \%)$ \\
\hline Low activity (2.6-3.2), $n$ (\%) & $1(3 \%)$ \\
\hline Medium activity (3.2-5.1), $n(\%)$ & $11(35 \%)$ \\
\hline High activity (>5.1), $n(\%)$ & $15(48 \%)$ \\
\hline CRP (mg/L), median (range) & $4.0(<0.5-106.2)$ \\
\hline ESR (mm/h), median (range) & $29(4-84)$ \\
\hline WBC $\left(\times 10^{9} / L\right)$ & $6.5(3.5-12.0)$ \\
\hline \multicolumn{2}{|l|}{ Medication, $n(\%)$} \\
\hline Glucocorticoids & $18(58 \%)$ \\
\hline DMARDs & $24(77 \%)$ \\
\hline
\end{tabular}

$R F$, rheumatoid factor; Anti-CCP, anti-cyclic citrullinated peptide; DAS28, disease activity score 28; CRP, C-reactive protein; ESR, erythrocyte sedimentation rate; WBC, white blood count; DMARDs, disease-modifying anti-rheumatic drugs. 
of the patient cohort ranged from remission (the disease activity score- 28 , DAS28 < 2.6) to high (DAS28 > 5.1), with the median value of 4.92 . Four naïve patients had no treatment history. The rest patients were treated with low dose of glucocorticoids and/or disease-modifying anti-rheumatic drugs. Those with a treatment history with high-dose glucocorticoids $(>10 \mathrm{mg} /$ day) or biologics within the past 6 months were excluded from the study.

\section{Increased Tfh Cell Differentiation in RA Patients Correlated Disease Activity}

$\mathrm{CD}^{+} \mathrm{T}$ cells play an important role in the pathogenesis of RA $(1-3,29)$ but very few efforts have investigated all major $\mathrm{CD} 4^{+} \mathrm{T}$ cell subsets in a single study. Using multicolor flow cytometry, the frequencies of Treg $\left(\mathrm{CD} 25^{\text {high }}\right)$ and conventional $\mathrm{CD} 4^{+}$subsets including naïve $\left(\mathrm{CD} 25^{-} \mathrm{CD} 45 \mathrm{RA}^{+} \mathrm{CD} 6 \mathrm{~L}^{+}\right)$, Th1 $\left(\mathrm{CD} 25^{-} \mathrm{CD} 45 \mathrm{RA}^{-}\right.$ $\left.\mathrm{CXCR}^{+}{ }^{+} \mathrm{CCR}^{-}{ }^{-} \mathrm{CCR} 4^{-}\right)$, Th2 (CD25-CD45RA-CXCR3-CCR6 ${ }^{-}$ $\left.\mathrm{CCR}^{+}\right)$, Th17 $\left(\mathrm{CD} 25^{-} \mathrm{CD} 45 \mathrm{RA}^{-} \mathrm{CXCR}^{-}{ }^{-} \mathrm{CCR} 6^{+} \mathrm{CCR} 4^{+}\right)$, and Tfh $\left(\mathrm{CD} 25^{-} \mathrm{CD} 45 \mathrm{RA}^{-} \mathrm{CXCR} 5^{+} \mathrm{PD}-1^{+}\right)$subsets in $\mathrm{CD} 4^{+} \mathrm{T}$ cells were analyzed simultaneously (30) (Figure 1A; Figure S1 in Supplementary Material). The comparison between these subsets in healthy individuals and RA patients revealed a significantly higher frequency of Tfh cells in RA patients, increasing from an average of $1.6 \%$ in the controls to $2.6 \%$ in RA patients (Figure 1B). On the contrary, we did not observe any significant difference for other $\mathrm{CD} 4^{+} \mathrm{T}$ cell subsets between healthy individuals and RA patients. We also examined the potential correlations between the frequencies of $\mathrm{CD}^{+} \mathrm{T}$ cell subsets and disease activities as measured by DAS28. Again, only Tfh cells' frequency, but not others' showed a positive correlation with DAS28 values $(p$-value $=0.005)($ Figure 2$)$. The increase of the Tfh activation and the correlation between the aberrant Tfh differentiation and RA disease activity support the notion that Tfh cells participate in the pathogenesis of RA.

\section{Hyperactivation of STAT3 in CD4 ${ }^{+}$T Cells Correlated With Tfh Differentiation in RA Patients}

The increase of Tfh cells in RA was reported in previous studies (15-19) and confirmed in our cohort. However, the reason that caused such immune dysregulation remained unknown. We hypothesized the constitutive activation of STAT3 in RA (25-28) could promote the Tfh differentiation since STAT3 is pivotal for the generation of Tfh cells (5). We took the advantage of Phosflow to quantify the phosphorylation of STAT3 in CD4 ${ }^{+}$ $\mathrm{T}$ cells. We could detect substantial expression of pSTAT3 in $\mathrm{CD}^{+} \mathrm{T}$ cells but not in B cells (Figure 3A). Importantly, the expression of pSTAT3 in CD4 ${ }^{+} \mathrm{T}$ cells from RA patients (mean value: $27.0 \%$ ) was 2.4 -fold higher than that of $\mathrm{CD}^{+} \mathrm{T}$ cells from healthy controls (mean value: $11.4 \%)(p=0.002)$ (Figure 3B). We did not detect any difference of pSTAT3 expression in B cells between the two groups. We further analyzed the expression on pSTAT3 in each CD4 ${ }^{+} \mathrm{T}$ cell subset as shown in Figure 1A. To our surprise, all $\mathrm{CD} 4^{+} \mathrm{T}$ cell subsets had significantly higher

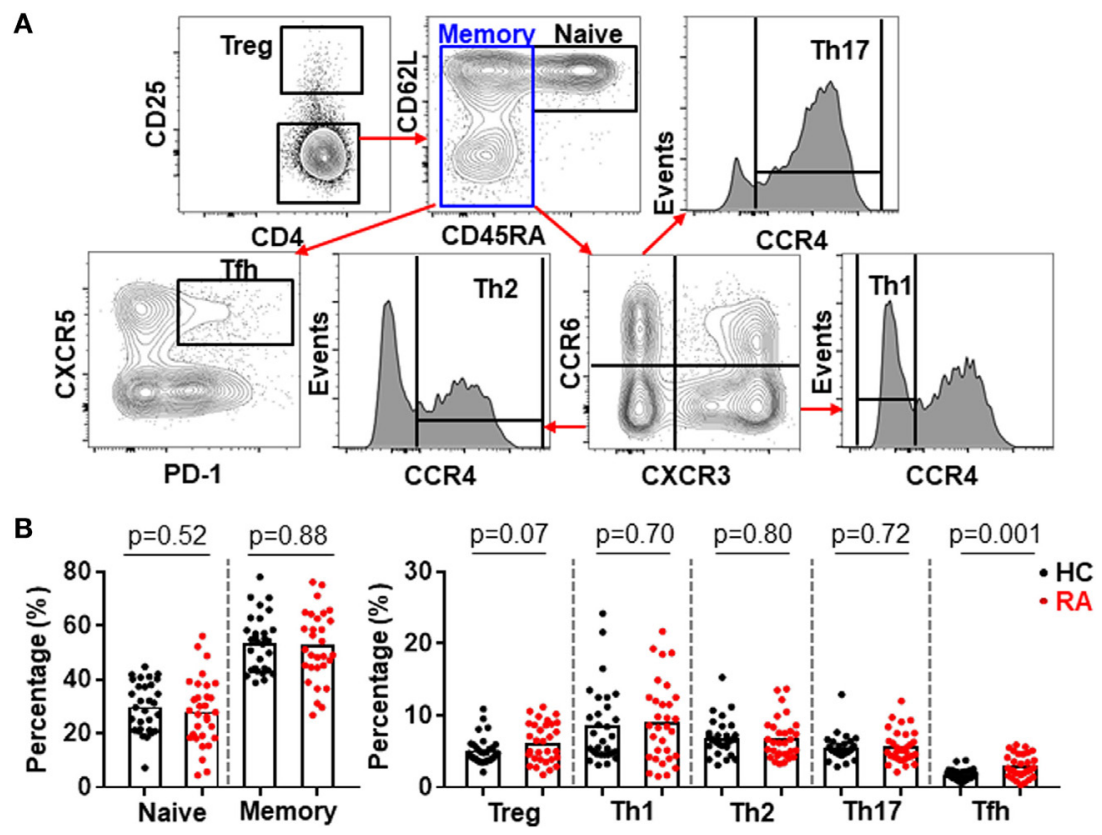

FIGURE 1 | Increased follicular helper T (Tfh) cell differentiation in patients with rheumatoid arthritis (RA). Peripheral blood mononuclear cells from RA patients and healthy control individuals $(\mathrm{HC})$ were analyzed by flow cytometry. (A) FACS plots showing the gating of $\mathrm{ZA}^{-} \mathrm{TCRab}^{+} \mathrm{CD} 4^{+}$viable $\mathrm{CD} 4^{+} \mathrm{T}$ cells for Treg (CD25 high) and

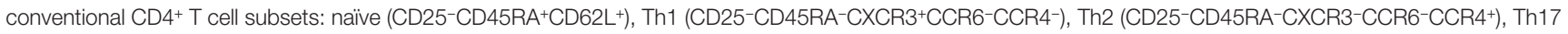
$\left(\mathrm{CD} 25^{-} \mathrm{CD} 45 \mathrm{RA}{ }^{-} \mathrm{CXCR} 3^{-} \mathrm{CCR} 6^{+} \mathrm{CCR} 4^{+}\right)$, and Tfh $\left(\mathrm{CD} 25^{-} \mathrm{CD} 45 \mathrm{RA}^{-} \mathrm{CXCR} 5^{+} \mathrm{PD}-1^{+}\right)$. (B) Statistics showing the percentages of $\mathrm{CD} 4^{+} \mathrm{T}^{-}$cell subsets in total $\mathrm{CD} 4^{+}$ T cells. Each dot represents the value of an individual subject with columns showing the mean values of each group. The $p$-values were obtained using Student's t-tests. 

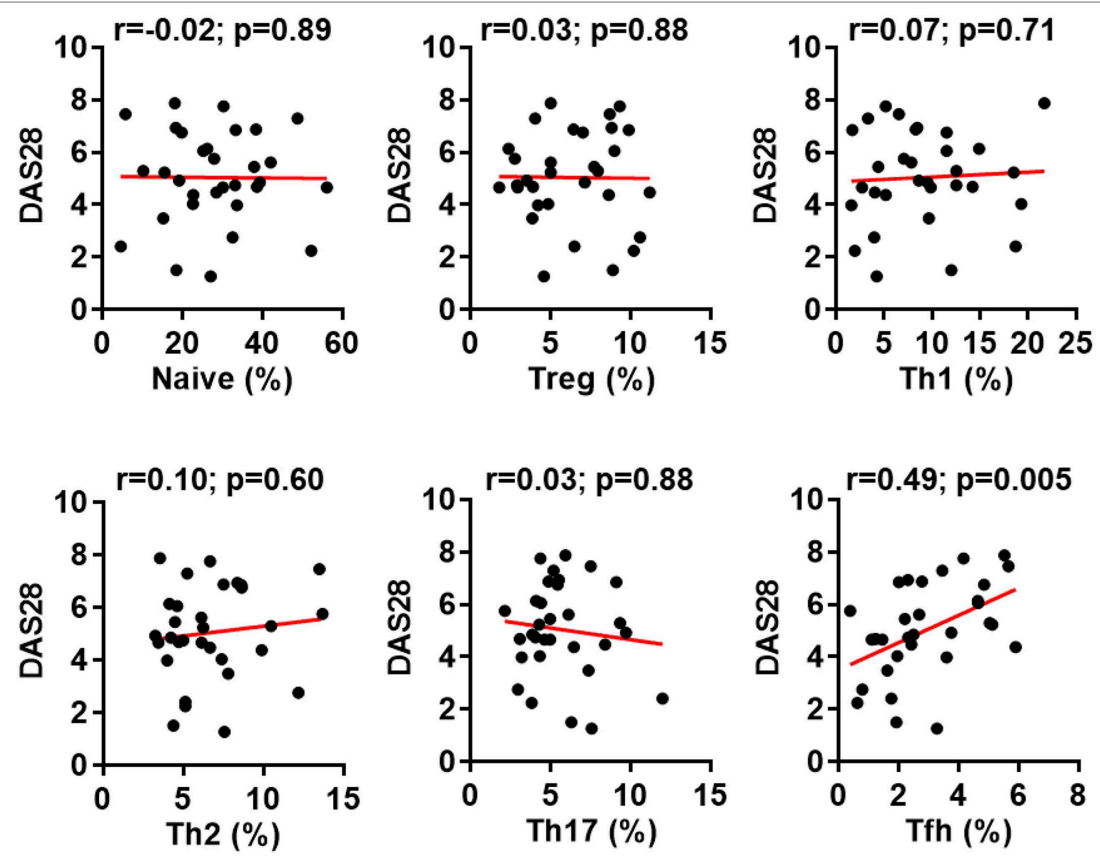

FIGURE 2 | Increased follicular helper T (Tfh) cell differentiation correlates with rheumatoid arthritis (RA) disease activity. The percentages of CD4+ T cell subsets in the peripheral blood mononuclear cells from patients with RA were analyzed as Figure 1. The correlation between the frequencies of these subsets and the disease activities measured by DAS28 were determined using Spearman's correlation coefficient.

level of pSTAT3 in RA patients than those in HC (Figure 3C). There was an approximate twofold increase of pSTAT3 in each of $\mathrm{CD}^{+} \mathrm{T}$ cell subsets in RA as compared to those in healthy controls (Figure 3D).

We then asked whether the phosphorylation of STAT3 associated with the generation of Tfh cells or other CD $4^{+}$ $\mathrm{T}$ cell subsets in RA. By analyzing the relationship between the pSTAT3 expression in total CD $4^{+} \mathrm{T}$ cells and the frequencies of $\mathrm{CD}^{+} \mathrm{T}$ cell subsets, we could only detect a modest but significant correlation between the status of STAT3 phosphorylation in $\mathrm{CD}^{+} \mathrm{T}$ cells and the $\mathrm{Tfh}$ generation $(p$-value $=0.047)$ (Figure 4A). Intriguingly, we also detected a specific correlation of between STAT3 phosphorylation in CD4 ${ }^{+} \mathrm{T}$ cells and Tfh frequencies in HC (Figure S2 in Supplementary Material). It was possible that the differentiation of each $\mathrm{CD} 4^{+} \mathrm{T}$ cells might be more specifically affected by the phosphorylation of STAT3 in their own. To test this, we performed a similar study to examine the relationship between the pSTAT3 expression in individual $\mathrm{CD}^{+} \mathrm{T}$ cell subset and their frequencies. The STAT3 phosphorylation showed no correlation with the frequencies of naïve, Treg, Th1, Th2, or Th17 cells. The correlation with Tfh cells, nevertheless, became more obvious $(p$-value $=0.009)($ Figure 4B). The results strongly suggest the hyperactivated STAT3 drove the Tfh differentiation in patients with RA.

In addition to dissecting a role of the STAT3 pathway in regulating $\mathrm{CD}^{+} \mathrm{T}$ cell differentiation, we also tested whether the status of STAT3 phosphorylation might affect the disease severity. We found a clear correlation between patients' disease activities and the pSTAT3 expression, not only in total CD4 ${ }^{+} \mathrm{T}$ cells
(Figure 5A) but also in each $\mathrm{CD}^{+} \mathrm{T}$ cell subsets (Figure 5B). This indicates the contribution of STAT3 hyperactivation to RA is not limited to a specific $\mathrm{CD}^{+} \mathrm{T}$ cell population, but rather broad.

\section{STAT3-Stimulating Cytokines Were Elevated in RA Patients}

Cytokines IL-6, IL-10, IL-21, and leptin can activate STAT3 phosphorylation in $\mathrm{CD}^{+} \mathrm{T}$ cells $(31,32)$. Could these cytokines induce the enhanced STAT3 phosphorylation in RA patients? We first measured the production of these cytokines in RA patients' plasma. Results by ELISA demonstrated upregulated production of all four cytokines with the most prominent increase for IL-6: a 7.3-fold upregulation from $6.6 \pm 1.1 \mathrm{pg} / \mathrm{mL}$ in healthy individuals to $48.06 \pm 8.9 \mathrm{pg} / \mathrm{mL}$ in RA patients (Figure 6A). The increase for IL-10, IL-21, and leptin was about twofold (Figures 6B-D). To define the contribution of the elevated pro-inflammatory cytokines to the hyperactivation of STAT3 and the disease activities, we analyzed the relationship between cytokine levels and the disease activity DAS28 or the pSTAT3 expression. IL-6 levels significantly correlated with DAS28 $(p$-value $=0.006)$ and pSTAT3 ( $p$-value $<0.001$ ) (Figure 7A). Other cytokines also showed general trends for positive correlations but not significant (Figures 7B-D). These results spotlight a central role of IL-6 in inducing pSTAT3 in CD4 ${ }^{+} \mathrm{T}$ cells and for the development of the disease. We also test whether there was a relationship between the Tfh frequency and any of these STAT3-stimulating cytokines but could not identify any substantial link. We thus conclude that although the Tfh differentiation in RA patients is critically 

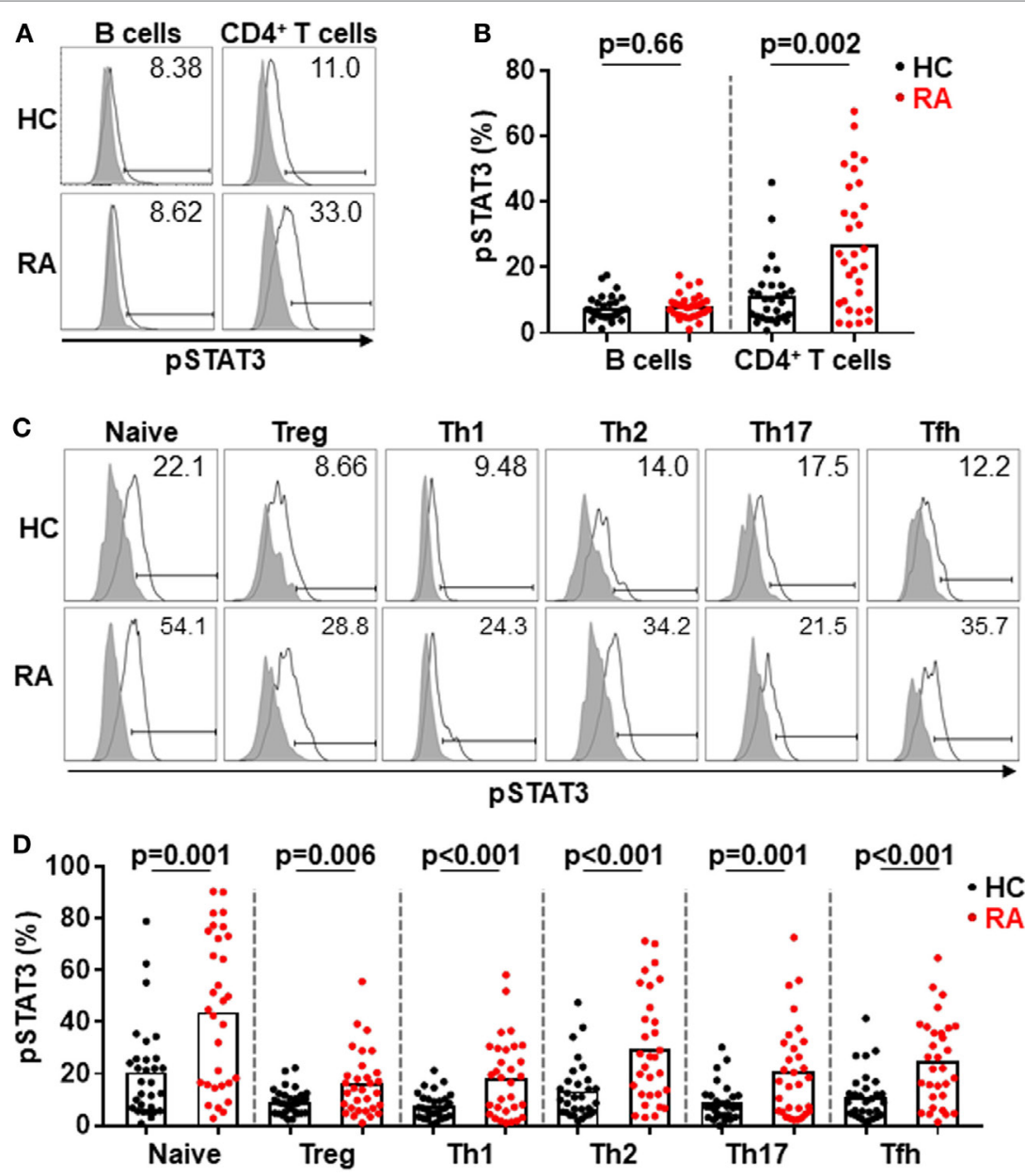

FIGURE 3 | Constitutive phosphorylation of signal transducer and activator of transcription 3 (STAT3) in CD4 ${ }^{+}$T cells from patients with rheumatoid arthritis (RA). The expression of intracellular phosphorylated STAT3 (pSTAT3) was analyzed using Phosflow assays in CD4+ $4^{+}$cells and B cells from RA patients. (A) FACS plots showing representative staining patterns for pSTAT3 (empty histograms) and an isotype control antibody (filled histograms) of indicated immune cell types; Numbers indicating the percentages of the pSTAT3 positive population. (B) Statistics showing the percentages of pSTAT3 positive population in total ZA-CD3-CD19+ viable $\mathrm{B}$ cells and ZA-TCRab+CD4+ viable CD4+ ${ }^{+}$cells from RA or HC groups. (C) FACS plots showing representative staining patterns for pSTAT3 and an isotype control antibody of indicated CD4+ T cell subsets; numbers indicating the percentages of the pSTAT3 positive population. (D) Statistics showing the percentages of pSTAT3 positive population in indicated CD4 ${ }^{+} \mathrm{T}$ cell subsets from RA or HC groups. Each dot represents the value of an individual subject with columns showing the mean value of each group. The $p$-values were obtained using Student's $t$-tests.

driven by the STAT3 phosphorylation, it may not depend on a single cytokine. A synergistic effect of multiple cytokines or other signals are candidate mechanisms.

\section{DISCUSSION}

$\mathrm{CD}^{+} \mathrm{T}$ cells have long been regarded as a key player in the pathogenesis of RA (29). Indeed, genetic variants in the major histocompatibility complex (MHC) region, especially MHC class II HLA-DRB1 genes contribute to the overall 11-37\% genetic risk of RA. Non-HLA genes encoding molecules directly involved in pathways of T-cell function including PTPN22, IL23R, CTLA4, STAT4, and CD40 are also ranked high in the identified RA-risk loci (33). Although early studies discovered synovial effector $\mathrm{CD}^{+} \mathrm{T}$ cells predominantly produce Th1 cytokines IFN $\gamma$ and
$\mathrm{TNF} \alpha$ (34), more recent research has been focusing on later discovered $\mathrm{CD}^{+} \mathrm{T}$ cell subsets: Th17 and Tfh cells.

In the cohort of RA patients we examined, there was a significant increase of CXCR $5^{+} \mathrm{PD}-1^{+}$memory Tfh cells in blood, as compared to that of healthy controls. The frequency of Tfh cells positively correlated with the disease activities, as measured by DAS28. This observation was largely in agreement with several published reports showing the aberrant function of Tfh cells in RA patients (15-19). The circulating memory Tfh cells can be analyzed in two different ways $(35,36)$. Studies using $\mathrm{CXCR}^{+} \mathrm{PD}-1^{+}$or $\mathrm{CXCR}^{+}{ }^{+} \mathrm{COS}^{+}$to mark Tfh cells indicated an active Tfh differentiation in RA $(15,16,18)$ while those applying CCR6 and CCR3 to stratify Tfh cells demonstrated a biased Tfh polarization into CXCR5 ${ }^{+} \mathrm{CCR} 6^{+} \mathrm{CXCR}^{-}{ }^{-}$(Th17type Tfh) and/or $\mathrm{CXCR}^{+} \mathrm{CCR} 6^{-} \mathrm{CXCR}^{-}$(Th2-type Tfh) 

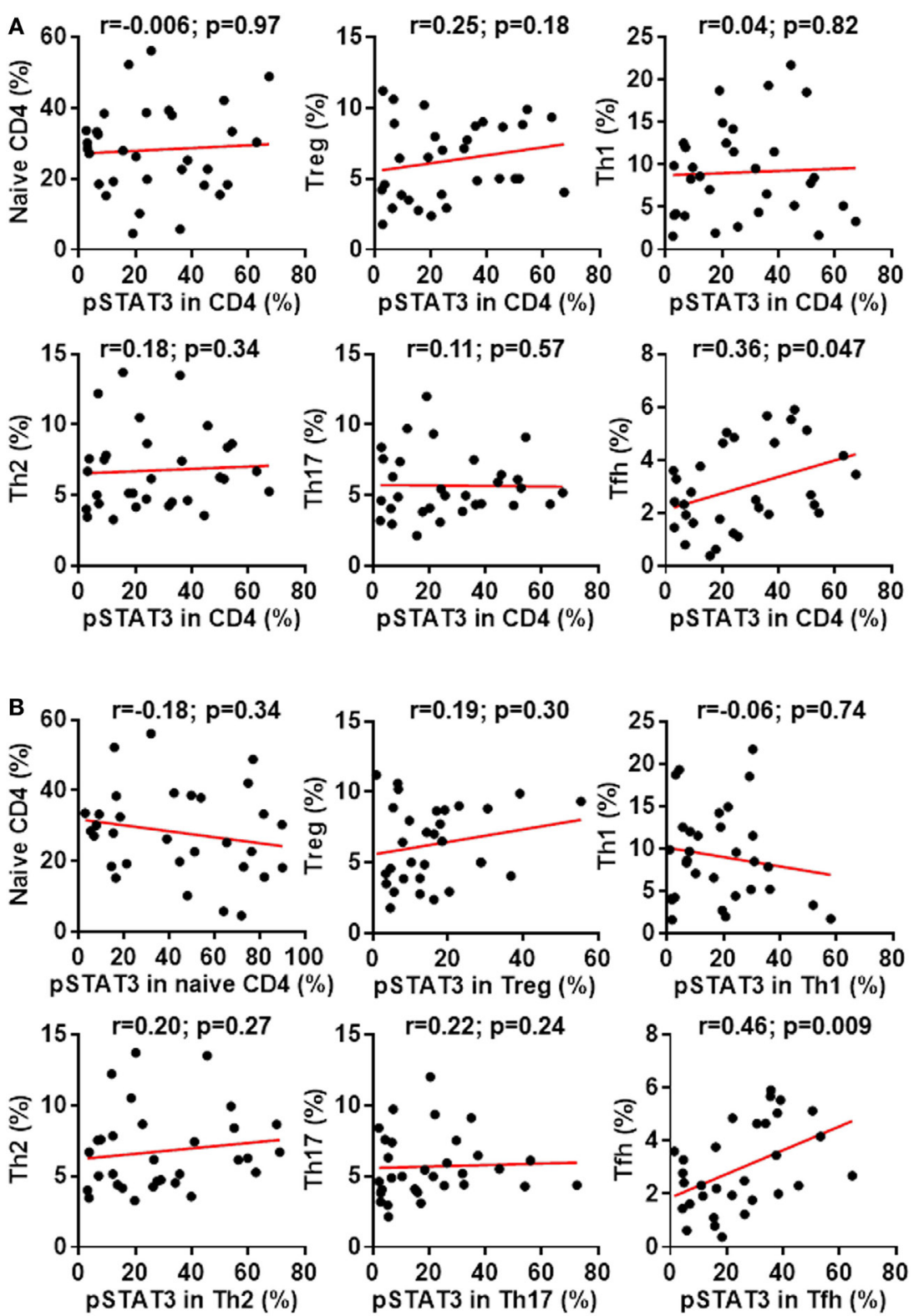

FIGURE 4 | Signal transducer and activator of transcription 3 (STAT3) hyperactivation correlates with aberrant follicular helper T (Tfh) differentiation in patients with rheumatoid arthritis (RA). Statistics showing the relationship between the frequencies of indicated CD4+ T cell subsets with the phosphorylated STAT3 (pSTAT3) expression in total CD4+ $\mathrm{T}$ cells $\mathbf{( A )}$ or in each individual subsets (B) in the peripheral blood mononuclear cells from patients with RA. The correlation was determined using Spearman's correlation coefficient.

subsets in RA patients $(18,19)$. Despite different analytic methodologies, such evidence strongly supports a significant contribution of Tfh cells to systemic inflammation and autoimmunity that drive the development of RA. To be noted, the phenotype of circulating Tfh cells is different from B-helper $\mathrm{T}$ cells infiltrated in inflammatory synovial tissues in RA. The latter $\mathrm{CD}^{+}{ }^{+} \mathrm{T}$ cell population, though expressing conventional Tfh markers including PD-1, ICOS, CXCL13, and IL-21, do not upregulate CXCR5 (37). It remains unclear whether these $\mathrm{B}$-helper T cells in joints also participate in systemic immune activation in RA.
There is a major question left unanswered by the studies that characterized Tfh cells in RA patients-what induces the aberrant Tfh differentiation? We set to test the hypothesis that the constitutive activation of STAT3 in RA led to enhanced Tfh differentiation. By measuring the phosphorylation of STAT3, we found a drastic increase of pSTAT 3 in $\mathrm{CD} 4^{+} \mathrm{T}$ cells but not in B cells. The increase of pSTAT3 was detected in all CD4 ${ }^{+}$ $\mathrm{T}$ cell subsets with highest phosphorylation status in naïve $\mathrm{CD} 4^{+} \mathrm{T}$ cells. In human $\mathrm{CD} 4^{+} \mathrm{T}$ cells, naïve $\mathrm{CD} 4^{+} \mathrm{T}$ cells were shown to express a lower level of suppressor of cytokine signaling 3 (SOCS3), which interacts with cytokine receptors and 

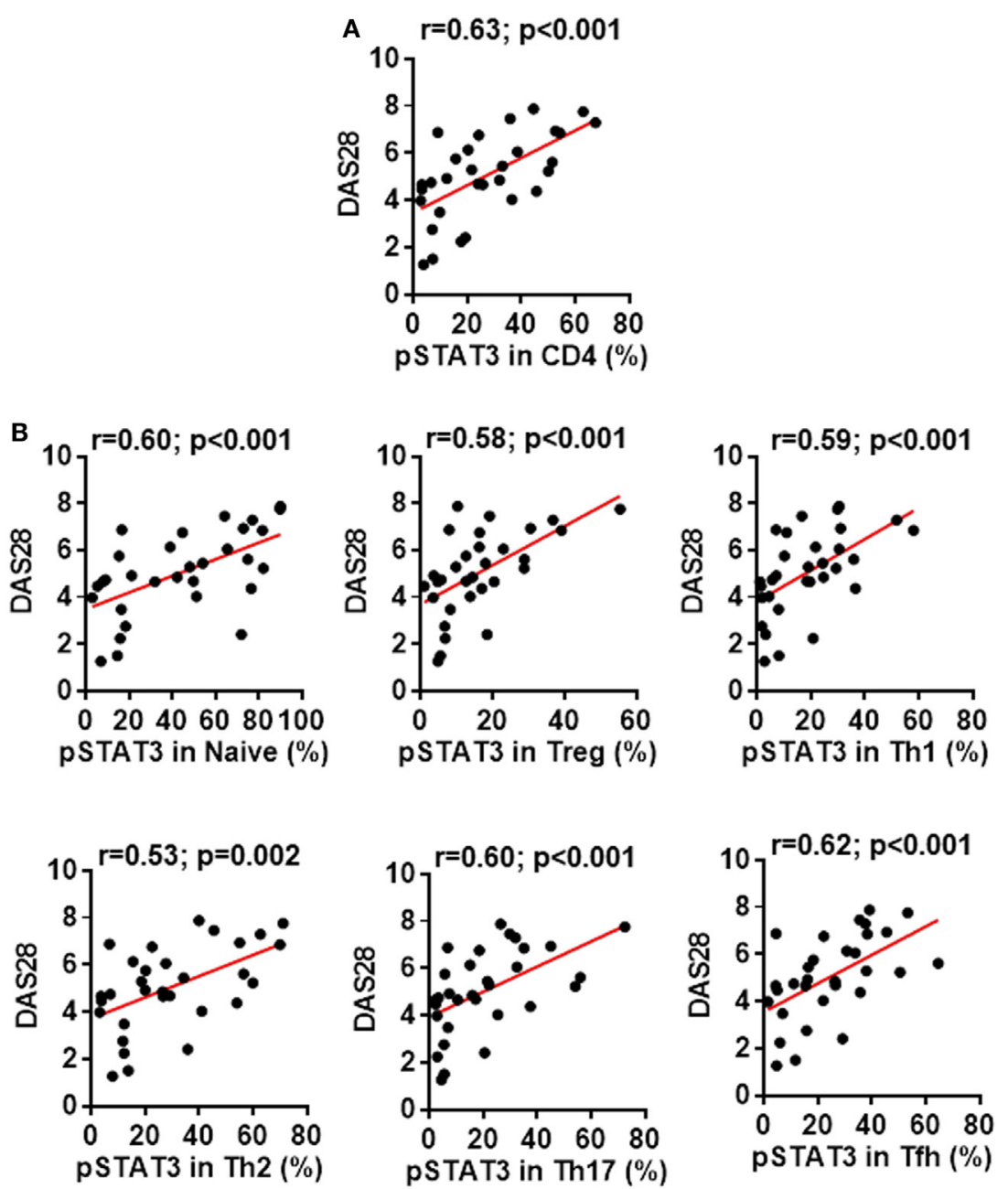

FIGURE 5 | Signal transducer and activator of transcription 3 (STAT3) hyperactivation correlates with rheumatoid arthritis (RA) disease activity. Statistics showing the relationship between the disease activities measured by DAS28 with the phosphorylated STAT3 (pSTAT3) expression in total CD4+ T cells (A) or in each individual subsets (B) in the peripheral blood mononuclear cells from patients with RA. The correlation was determined using Spearman's correlation coefficient.

inhibits receptor-mediated signal transduction (38). As the induction of SOCS3 represents the key mechanism to block the activation of STAT3 (39), the low expression of SOCS3 may lead to a high pSTAT3 in naïve $\mathrm{CD} 4^{+} \mathrm{T}$ cells. For effector $\mathrm{CD}^{+} \mathrm{T}$ cell subsets, pSTAT3 was detected lowest in Th1 cells, reflecting the fact that STAT3 functions to promote Th2, Th17, and Tfh differentiation but inhibit Th1 differentiation (22, 23, 39-43). Despite the high levels of pSTAT3 in all three subsets, the intensity of pSTAT3 in $\mathrm{CD} 4^{+} \mathrm{T}$ cells only correlated with the frequency of Tfh cells but not Th2 or Th17 cells, suggesting redundant factors other than STAT3 potently regulate Th2 and Th17 differentiation. The key role of pSTAT3 in promoting the Tfh generation was further recognized by a strong correlation between the frequency of Tfh cells and the pSTAT3 expression in Tfh cells.

Since the STAT3 hyperactivation was observed across all CD4 ${ }^{+}$ $\mathrm{T}$ cells, we believe it was caused a systemic change of the immune system that is not specific for certain subsets. We measured the serum concentrations of cytokines IL-6, IL-10, IL-21, and leptin, all of which have been shown to induce the phosphorylation of STAT3 in T cells $(31,32)$. All of these cytokines were upregulated in RA patients as compared to healthy controls, with a prominent increase of IL- 6 for more than sevenfold. By analyzing the correlation between the plasma cytokine concentrations and the pSTAT3 status in CD4 ${ }^{+}$T cells, IL- 6 appears to play a central role in determining the activation of STAT3 in $\mathrm{CD}^{+}$ $\mathrm{T}$ cells. Other cytokines may synergistically enhance pSTAT3 as there were general trends for positive correlations. It has been previously shown that serum IL-21 concentrations modestly correlated with the frequencies of Tfh cells in RA patients (44). In our cohort, we also observed a similar trend of positive correlation between IL-21 concentration and Tfh activation, but it was not statistically significant $(p$-value $=0.06)$. There were no significant correlation between the frequency of Tfh cells and the concentration of any of tested STAT3-activating cytokines. It could be well explained by the fact that the Tfh differentiation is 

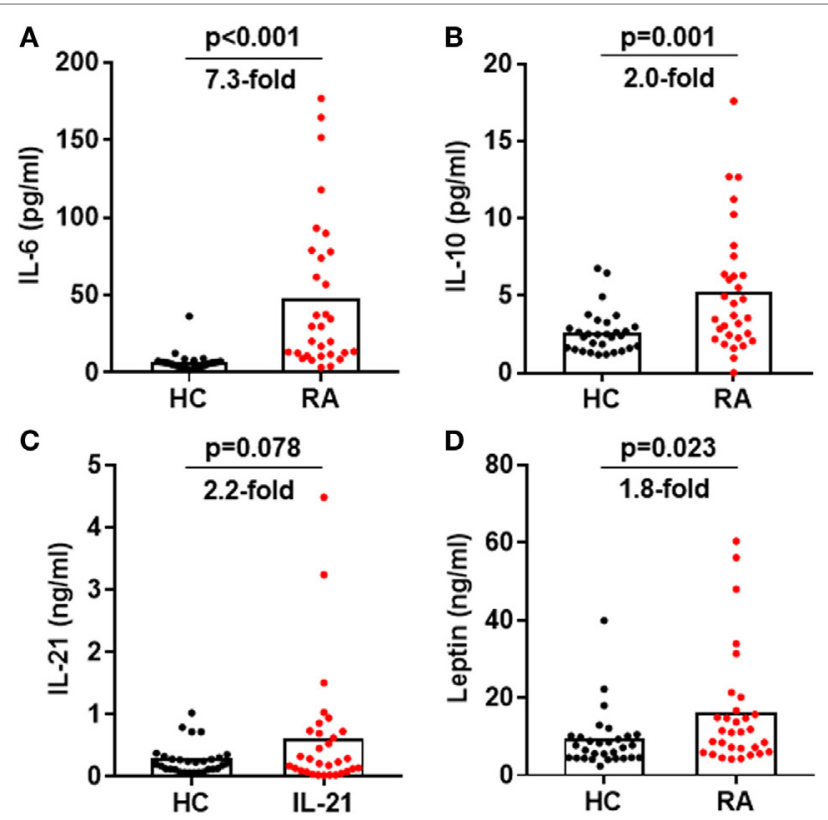

FIGURE 6 | Increased signal transducer and activator of transcription 3-stimulating cytokines in the plasma of rheumatoid arthritis (RA) patients. Plasma was isolated from blood from RA patients and healthy controls. The amount of IL-6 (A), IL-10 (B), IL-21 (C), and leptin (D) was measured by ELISA. Each dot represents the value of an individual subject with columns showing the mean values of each group. The $p$-values were obtained using Student' $t$-tests.

induced by the combination of multiple cytokines (20). It is also possible that cytokines may preferably execute in supporting Tfh differentiation in local lymphoid tissue spleen and lymph nodes so the plasma concentrations were not always representative of its tissue concentrations.

Our study revealed an IL-6-pSTAT3-Tfh axis in regulating immune responses in RA patients. As the status of each of three components individually correlated with the disease activity indicator DAS 28, this immunoregulatory axis is likely to reside proximal to the center of RA pathogenesis. Therapeutics have been developed to target this axis including monoclonal antibodies to block IL- 6 and small molecules to inhibit Janus kinases (JAKs) that act upstream of the STAT3 pathway. IL-6 blockers such as tocilizumab and JAK inhibitors such as tofacitinib have demonstrated profound efficacies and been approved to treat RA (45). Intriguingly, tocilizumab therapy was shown not only reducing pSTAT3 in $\mathrm{CD}^{+} \mathrm{T}$ cells (46) but also inhibiting the Tfh differentiation (47). The evidence gained here and those from previous studies justify targeting the IL-6-pSTAT3-Tfh axis for biomarker discovery and drug development for RA diagnosis and therapy.

\section{MATERIALS AND METHODS}

\section{Study Subjects}

Thirty-one patients diagnosed as RA according to the $2010 \mathrm{RA}$ classification criteria by American College of Rheumatology/
European League Against Rheumatism (48) were recruited. The cohort included both newly diagnosed patients $(n=4)$ and patients with long-standing disease $(n=27)$. No patients had a history of treatment with biologic agents or high-dose corticosteroids (>10 mg/day) 6 months preceding the study. Thirty healthy individuals with comparable demographics were recruited as controls. All participants consented in writing to donate blood for the study. Ethics was approved by human ethics committees of Renji Hospital and Ruijin Hospital affiliated to Shanghai Jiao Tong University School of Medicine (Shanghai, China) and Affiliated Hospital of Hubei University for Nationalities (Hubei, China).

\section{Isolation of Peripheral Blood Mononuclear Cells (PBMCs) and Plasma}

Blood from healthy individuals and patients with RA were collected in BD Vacutainer ${ }^{\circledR}$ Blood Collection Tubes (BD). After the centrifugation $\left(300 \mathrm{~g}, 20^{\circ} \mathrm{C}, 5 \mathrm{~min}\right)$, plasma was collected and stored in $-80^{\circ} \mathrm{C}$ for further analysis. Cells were diluted with PBS (1:1), and gently loaded to Ficoll-Paque Plus (GE) layer at the ratio of 1:1 (PBS + blood cells:Ficoll), followed by density gradient centrifugation $\left(450 \mathrm{~g}, 20^{\circ} \mathrm{C}, 20 \mathrm{~min}\right.$, no brake). Mononuclear cell layer was transferred to a new tube and washed with cold PBS. Cells were suspended in cold FACS buffer (1\% BSA, 0.05\% NaN3) for further analysis.

\section{Flow Cytometric Analysis}

Freshly isolated PBMCs were incubated with following fluorochrome-conjugated monoclonal antibodies in FACS buffer for surface staining. Antibodies (from BD Biosciences and BioLegend) were TCR $\alpha \beta$ (clone IP26), CXCR3 (clone G025H7), CD45RA (clone HI100), CD3 (clone UCHT1), PD-1 (clone EH12.2H7), CD19 (clone SJ25C1), CCR6 (clone 11A9), CD8a (clone SK1), CCR4 (clone L291H4), CD62L (clone DREG-56), CD25 (clone BC96), CXCR5 (clone RF8B2), CD4 (clone RPAT4), and dead cells stained with Zombie Aqua were excluded from analysis. After the surface staining, cells were washed with FACS buffer and then fixed with pre-warmed Phosflow ${ }^{\mathrm{TM}}$ Fix Buffer I (BD) in $37^{\circ} \mathrm{C}$ for $15 \mathrm{~min}$. Cells were washed and resuspended in pre-chilled Phosflow Perm Buffer III (BD) in $4^{\circ} \mathrm{C}$ for $25 \mathrm{~min}$. After wash, cells were stained with anti-pSTAT3 (BD, clone 4/ pSTAT3) at $37^{\circ} \mathrm{C}$ for $30 \mathrm{~min}$ to detect pSTAT3 (pY705). The same procedure was performed to stain with an isotype control antibody (BD, clone G155-178). The expression of surface markers and intracellular pSTAT3 were analyzed by a FACS analyser (LSRFortessa X-20, BD). The results were analyzed with FlowJo software (TreeStar).

\section{ELISA}

Cytokines in plasma samples were measured by ELISA kits (Biolegend: IL-6 and IL-21; Invitrogen: IL-10; R\&D systems: leptin) following the manufacturers' protocol. Plasma was diluted at 1:5 for IL-6, IL-10, and IL-21 and 1:100 for leptin, using ELISA diluent ( $1 \%$ BSA in PBS-T). Standards and diluted-plasma samples were incubated at room temperature for $2 \mathrm{~h}$, followed by incubations with detecting antibodies 

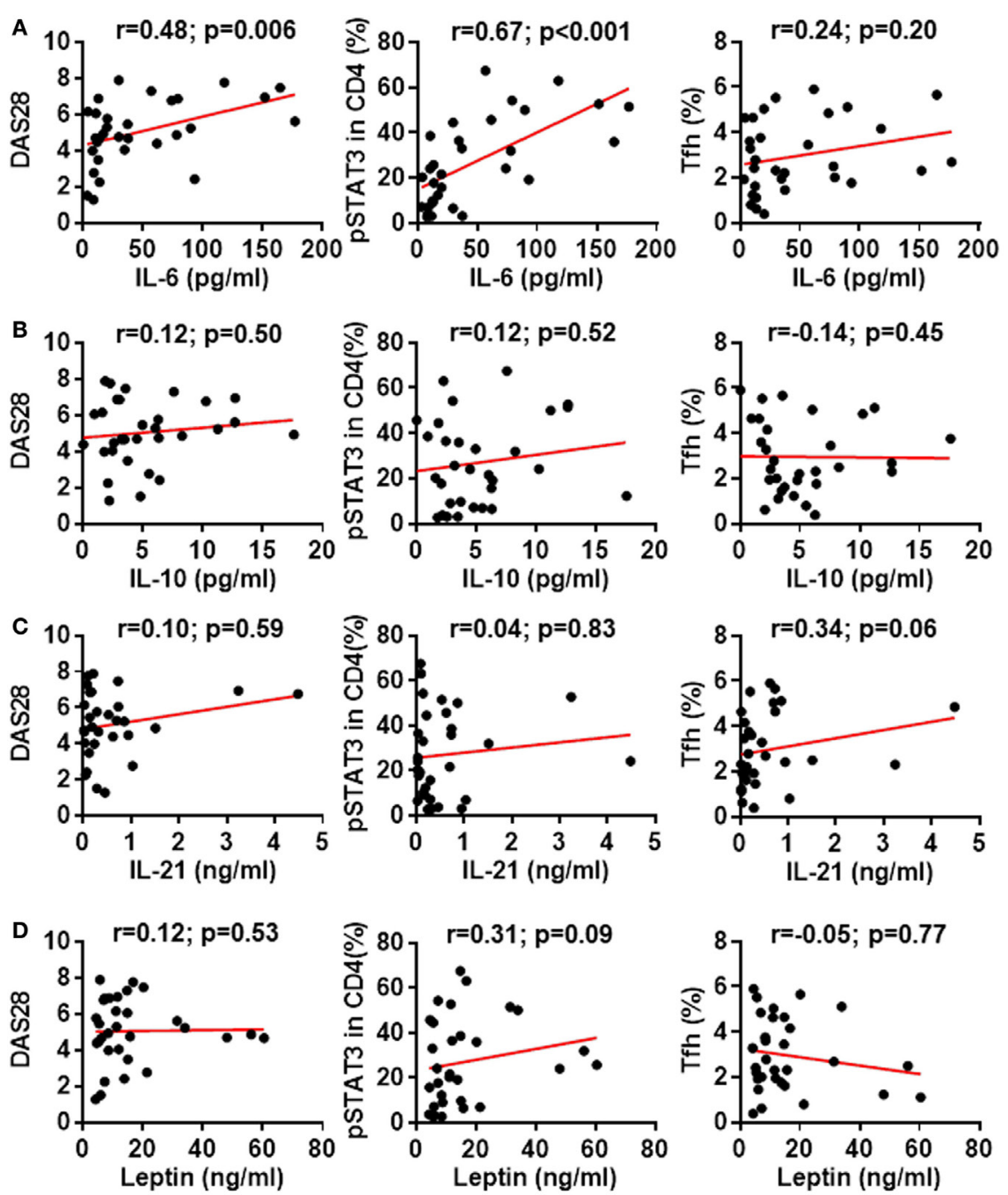

FIGURE 7 | Association between signal transducer and activator of transcription 3 (STAT3)-stimulating cytokines with STAT3 phosphorylation, disease activity or follicular helper T (Tfh) differentiation. Statistics showing the relationship between the expression of phosphorylated STAT3 (pSTAT3) in total CD4+ T cells, the disease activities measured by DAS28 or the frequency of Tfh cells with the amount of plasma IL-6 (A), IL-10 (B), IL-21 (C), or leptin (D). The correlation was determined using Spearman's correlation coefficient.

and streptavidin-horseradish peroxidase. The substrate $3,3^{\prime}, 5,5^{\prime}$-Tetramethylbenzidine was added before the values of optical densities were obtained by a microplate reader (SpectraMax 190, Molecular Devices).

\section{Quantitative PCR}

Naive (CD25-CD45RA ${ }^{+}{ }^{-}$D62 $\left.\mathrm{L}^{+}\right)$, Th1 $\left(\mathrm{CD} 25^{-} \mathrm{CD}^{-} 4 \mathrm{RA}^{-} \mathrm{CXCR}^{+}\right.$ $\left.\mathrm{CCR}^{-}{ }^{-} \mathrm{CCR} 4^{-}\right)$, Th2 $\left(\mathrm{CD} 25^{-} \mathrm{CD} 45 \mathrm{RA}^{-}{ }^{-} \mathrm{CXCR} 3^{-} \mathrm{CCR} 6^{-} \mathrm{CCR} 4^{+}\right)$, and Th17 (CD25- ${ }^{-}$D45RA $\left.{ }^{-} \mathrm{CXCR} 3^{-} \mathrm{CCR} 6^{+} \mathrm{CCR} 4^{+}\right)$cells were sorted with a FACS cell sorter (BD Aria III). mRNA samples were extracted with Trizol reagents, and cDNA were synthesized with cDNA Synthesis Kit (Takara). Following, Tbx21 forward 5'-ATTGCCG TGACTGCCTACCAGA-3' and reverse $5^{\prime}$-GGAATTGACAGT TGGGTCCAGG-3'; Gata3 forward 5' -ACCACAACCACACTC TGGAGGA- ${ }^{\prime}$ and reverse $5^{\prime}$-TCGGTTTCTGGTCTGGATG CCT-3'; Rorc forward 5'-GAGGAAGTGACTGGCTACCAGA-3' and reverse 5'-GCACAATCTGGTCATTCTGGCAG-3'; Ifng forward $5^{\prime}$-GAGTGTGGAGACCATCAAGGAAG-3' and reverse 5'-TGCTTTGCGTTGGACATTCAAGTC-3'; Il4 forward 5' -CC GTAACAGACATCTTTGCTGCC-3' and reverse 5'-GAGTGTC

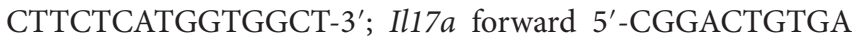
TGGTCAACCTGA-3' and reverse 5'-GCACTTTGCCTCCCA GATCACA-3'; Gapdh forward 5'-GTCTCCTCTGACTTCAAC

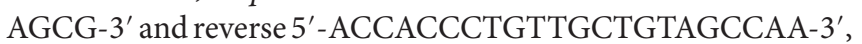
were used to measure the transcript levels using SYBR (Takara) with a Real-Time PCR Systems (QuantStudio 7 Flex, ABI). Relative fold change of gene expression was calculated by $2^{-(\mathrm{DCT} \text { experiment-DCT control) }} . \mathrm{DCT}=\mathrm{CT}^{\text {gene of interest }}-\mathrm{CT}^{\mathrm{GAPDH}}$.

\section{Statistical Analysis}

Data were analyzed with GraphPad Prism (version 7.0, GraphPad Software). The correlations of indicated parameters were 
determined by Spearman's correlation coefficient. Two-tailed $t$-tests were used to compare parameters between healthy individuals and RA patients. Results were considered statistically significant when $p$-values $<0.05$.

\section{ETHICS STATEMENT}

Ethics was approved by human ethics committees of Renji Hospital and Ruijin Hospital affiliated to Shanghai Jiao Tong University School of Medicine (Shanghai, China) and Affiliated Hospital of Hubei University for Nationalities (Hubei, China).

\section{AUTHOR CONTRIBUTIONS}

DY, JD, XC, and LLu conceived the study. DY, JD, and XG designed the experiments. XC, LLu, CF, RG, JF, YX, SX, SG, LY, WeiS, WenyanS, LLin, TJ, and DH helped to recruit patients and collect samples. JD, XG, QZ, DG, ZC, YC, and YW performed experiments and collected data. JD, XG, CF, LLu, XC, and DY interpreted the data and wrote the manuscript. All authors approved the final version.

\section{REFERENCES}

1. Smolen JS, Aletaha D, McInnes IB. Rheumatoid arthritis. Lancet (2016) 388:2023-38. doi:10.1016/S0140-6736(16)30173-8

2. Malmstrom V, Catrina AI, Klareskog L. The immunopathogenesis of seropositive rheumatoid arthritis: from triggering to targeting. Nat Rev Immunol (2017) 17:60-75. doi:10.1038/nri.2016.124

3. McInnes IB, Schett G. Cytokines in the pathogenesis of rheumatoid arthritis. Nat Rev Immunol (2007) 7:429-42. doi:10.1038/nri2094

4. Mankia K, Emery P. Preclinical rheumatoid arthritis: progress toward prevention. Arthritis Rheumatol (2016) 68:779-88. doi:10.1002/art.39603

5. Vinuesa CG, Linterman MA, Yu D, MacLennan IC. Follicular helper T cells. Annu Rev Immunol (2016) 34:335-68. doi:10.1146/annurev-immunol-041015055605

6. Linterman MA, Rigby RJ, Wong RK, Yu D, Brink R, Cannons JL, et al. Follicular helper $\mathrm{T}$ cells are required for systemic autoimmunity. J Exp Med (2009) 206:561-76. doi:10.1084/jem.20081886

7. Yu D, Vinuesa CG. Multiple checkpoints keep follicular helper T cells under control to prevent autoimmunity. Cell Mol Immunol (2010) 7:198-203. doi:10.1038/cmi.2010.18

8. Craft JE. Follicular helper T cells in immunity and systemic autoimmunity. Nat Rev Rheumatol (2012) 8:337-47. doi:10.1038/nrrheum.2012.58

9. Zhong MC, Veillette A. The adaptor molecule signaling lymphocytic activation molecule (SLAM)-associated protein (SAP) is essential in mechanisms involving the Fyn tyrosine kinase for induction and progression of collagen-induced arthritis. J Biol Chem (2013) 288:31423-36. doi:10.1074/ jbc.M113.473736

10. Moschovakis GL, Bubke A, Friedrichsen M, Falk CS, Feederle R, Forster R. T cell specific Cxcr5 deficiency prevents rheumatoid arthritis. Sci Rep (2017) 7:8933. doi:10.1038/s41598-017-08935-6

11. Victoratos P, Kollias G. Induction of autoantibody-mediated spontaneous arthritis critically depends on follicular dendritic cells. Immunity (2009) 30:130-42. doi:10.1016/j.immuni.2008.10.019

12. Block KE, Zheng Z, Dent AL, Kee BL, Huang H. Gut microbiota regulates $\mathrm{K} / \mathrm{BxN}$ autoimmune arthritis through follicular helper $\mathrm{T}$ but not Th17 cells. J Immunol (2016) 196:1550-7. doi:10.4049/jimmunol.1501904

13. Chevalier N, Macia L, Tan JK, Mason LJ, Robert R, Thorburn AN, et al. The role of follicular helper $\mathrm{T}$ cell molecules and environmental influences in autoantibody production and progression to inflammatory arthritis in mice. Arthritis Rheumatol (2016) 68:1026-38. doi:10.1002/art.39481

\section{FUNDING}

The authors thank the funding supports by the National Key Research and Development Program of China (2017YFC0909003 to DY), National Basic Research Program of China 973 Program (2014CB541903 to DY), National Natural Science Foundation of China (31600708 to JD, 81429003 to DY, 81771729 to XC), the Australian National Health and Medical Research Council (GNT1147769 to DY), the Special Foundation of Taishan Overseas Distinguished Experts and Scholars (DY), the Shandong Provincial Natural Science Foundation, China (ZR2016YL013 to DY, ZR2015YL005 to DY and YW), the Priority Research Program of the Shandong Academy of Sciences (DY and YW). DY is supported by the Bellberry-Viertel Senior Medical Research Fellowship and intramural funds from Renji Hospital and The Australian National University.

\section{SUPPLEMENTARY MATERIAL}

The Supplementary Material for this article can be found online at https://www.frontiersin.org/articles/10.3389/fimmu.2018.01226/ full\#supplementary-material.

14. Teng F, Klinger CN, Felix KM, Bradley CP, Wu E, Tran NL, et al. Gut microbiota drive autoimmune arthritis by promoting differentiation and migration of peyer's patch T follicular helper cells. Immunity (2016) 44:875-88. doi:10.1016/ j.immuni.2016.03.013

15. Ma J, Zhu C, Ma B, Tian J, Baidoo SE, Mao C, et al. Increased frequency of circulating follicular helper T cells in patients with rheumatoid arthritis. Clin Dev Immunol (2012) 2012:827480. doi:10.1155/2012/827480

16. He J, Tsai LM, Leong YA, Hu X, Ma CS, Chevalier N, et al. Circulating precursor CCR7(lo)PD-1(hi) CXCR5(+) CD4(+) T cells indicate Tfh cell activity and promote antibody responses upon antigen reexposure. Immunity (2013) 39:770-81. doi:10.1016/j.immuni.2013.09.007

17. Wang J, Shan Y, Jiang Z, Feng J, Li C, Ma L, et al. High frequencies of activated $\mathrm{B}$ cells and $\mathrm{T}$ follicular helper cells are correlated with disease activity in patients with new-onset rheumatoid arthritis. Clin Exp Immunol (2013) 174:212-20. doi:10.1111/cei.12162

18. Arroyo-Villa I, Bautista-Caro MB, Balsa A, Aguado-Acin P, Bonilla-Hernan MG, Plasencia C, et al. Constitutively altered frequencies of circulating follicullar helper T cell counterparts and their subsets in rheumatoid arthritis. Arthritis Res Ther (2014) 16:500. doi:10.1186/s13075-014-0500-6

19. Singh D, Henkel M, Sendon B, Feng J, Fabio A, Metes D, et al. Analysis of CXCR5(+)Th17 cells in relation to disease activity and TNF inhibitor therapy in rheumatoid arthritis. Sci Rep (2016) 6:39474. doi:10.1038/srep39474

20. Ueno H, Banchereau J, Vinuesa CG. Pathophysiology of T follicular helper cells in humans and mice. Nat Immunol (2015) 16:142-52. doi:10.1038/ni.3054

21. Locci M, Wu JE, Arumemi F, Mikulski Z, Dahlberg C, Miller AT, et al. Activin A programs the differentiation of human TFH cells. Nat Immunol (2016) 17:976-84. doi:10.1038/ni.3494

22. Ray JP, Marshall HD, Laidlaw BJ, Staron MM, Kaech SM, Craft J. Transcription factor STAT3 and type I interferons are corepressive insulators for differentiation of follicular helper and T helper 1 cells. Immunity (2014) 40:367-77. doi:10.1016/j.immuni.2014.02.005

23. Ma CS, Avery DT, Chan A, Batten M, Bustamante J, Boisson-Dupuis S, et al. Functional STAT3 deficiency compromises the generation of human T follicular helper cells. Blood (2012) 119:3997-4008. doi:10.1182/blood2011-11-392985

24. Flanagan SE, Haapaniemi E, Russell MA, Caswell R, Allen HL, De Franco E, et al. Activating germline mutations in STAT3 cause early-onset multi-organ autoimmune disease. Nat Genet (2014) 46:812-4. doi:10.1038/ng.3040

25. Pratt AG, Swan DC, Richardson S, Wilson G, Hilkens CM, Young DA, et al. A CD4 $\mathrm{T}$ cell gene signature for early rheumatoid arthritis implicates 
interleukin 6-mediated STAT3 signalling, particularly in anti-citrullinated peptide antibody-negative disease. Ann Rheum Dis (2012) 71:1374-81. doi:10.1136/annrheumdis-2011-200968

26. Isomaki P, Junttila I, Vidqvist KL, Korpela M, Silvennoinen O. The activity of JAK-STAT pathways in rheumatoid arthritis: constitutive activation of STAT3 correlates with interleukin 6 levels. Rheumatology (Oxford) (2015) 54:1103-13. doi:10.1093/rheumatology/keu430

27. Kuuliala K, Kuuliala A, Koivuniemi R, Oksanen S, Hamalainen M, Moilanen E, et al. Constitutive STAT3 phosphorylation in circulating CD4+ T lymphocytes associates with disease activity and treatment response in recent-onset rheumatoid arthritis. PLoS One (2015) 10:e0137385. doi:10.1371/journal. pone. 0137385

28. Anderson AE, Pratt AG, Sedhom MA, Doran JP, Routledge C, Hargreaves B, et al. IL-6-driven STAT signalling in circulating CD4+ lymphocytes is a marker for early anticitrullinated peptide antibody-negative rheumatoid arthritis. Ann Rheum Dis (2016) 75:466-73. doi:10.1136/annrheumdis-2014-205850

29. Cope AP. T cells in rheumatoid arthritis. Arthritis Res Ther (2008) 10(Suppl 1): S1. doi:10.1186/ar2412

30. He J, Zhang X, Wei Y, Sun X, Chen Y, Deng J, et al. Low-dose interleukin-2 treatment selectively modulates $\mathrm{CD} 4(+) \mathrm{T}$ cell subsets in patients with systemic lupus erythematosus. Nat Med (2016) 22:991-3. doi:10.1038/nm.4148

31. O'Shea JJ, Schwartz DM, Villarino AV, Gadina M, McInnes IB, Laurence A. The JAK-STAT pathway: impact on human disease and therapeutic intervention. Annu Rev Med (2015) 66:311-28. doi:10.1146/annurev-med-051113-024537

32. Naylor C, Petri WA Jr. Leptin regulation of immune responses. Trends Mol Med (2016) 22:88-98. doi:10.1016/j.molmed.2015.12.001

33. Kim K, Bang SY, Lee HS, Bae SC. Update on the genetic architecture of rheumatoid arthritis. Nat Rev Rheumatol (2017) 13:13-24. doi:10.1038/ nrrheum.2016.176

34. Firestein GS. Evolving concepts of rheumatoid arthritis. Nature (2003) 423:356-61. doi:10.1038/nature01661

35. Schmitt N, Bentebibel SE, Ueno H. Phenotype and functions of memory Tfh cells in human blood. Trends Immunol (2014) 35:436-42. doi:10.1016/j.it.2014.06.002

36. Tsai LM, Yu D. Follicular helper T-cell memory: establishing new frontiers during antibody response. Immunol Cell Biol (2014) 92:57-63. doi:10.1038/ icb.2013.68

37. Rao DA, Gurish MF, Marshall JL, Slowikowski K, Fonseka CY, Liu Y, et al. Pathologically expanded peripheral $\mathrm{T}$ helper cell subset drives $\mathrm{B}$ cells in rheumatoid arthritis. Nature (2017) 542:110-4. doi:10.1038/nature20810

38. Kleinsteuber K, Heesch K, Schattling S, Sander-Juelch C, Mock U, Riecken K, et al. SOCS3 promotes interleukin-17 expression of human T cells. Blood (2012) 120:4374-82. doi:10.1182/blood-2011-11-392738

39. Hillmer EJ, Zhang H, Li HS, Watowich SS. STAT3 signaling in immunity. Cytokine Growth Factor Rev (2016) 31:1-15. doi:10.1016/j.cytogfr. 2016.05.001
40. Takeda K, Clausen BE, Kaisho T, Tsujimura T, Terada N, Forster I, et al. Enhanced Th1 activity and development of chronic enterocolitis in mice devoid of Stat3 in macrophages and neutrophils. Immunity (1999) 10:39-49. doi:10.1016/S1074-7613(00)80005-9

41. Yang XO, Panopoulos AD, Nurieva R, Chang SH, Wang D, Watowich SS, et al. STAT3 regulates cytokine-mediated generation of inflammatory helper T cells. J Biol Chem (2007) 282:9358-63. doi:10.1074/jbc.C600321200

42. Zhou L, Ivanov II, Spolski R, Min R, Shenderov K, Egawa T, et al. IL-6 programs $\mathrm{T}(\mathrm{H})-17$ cell differentiation by promoting sequential engagement of the IL-21 and IL-23 pathways. Nat Immunol (2007) 8:967-74. doi:10.1038/ nil 488

43. Stritesky GL, Muthukrishnan R, Sehra S, Goswami R, Pham D, Travers J, et al. The transcription factor STAT3 is required for T helper 2 cell development. Immunity (2011) 34:39-49. doi:10.1016/j.immuni.2010.12.013

44. Liu R, Wu Q, Su D, Che N, Chen H, Geng L, et al. A regulatory effect of IL-21 on $\mathrm{T}$ follicular helper-like cell and $\mathrm{B}$ cell in rheumatoid arthritis. Arthritis Res Ther (2012) 14:R255. doi:10.1186/ar4100

45. Misra DP, Agarwal V, Sharma A, Wakhlu A, Negi VS. 2016 update of the EULAR recommendations for the management of rheumatoid arthritis: a Utopia beyond patients in low/middle income countries? Ann Rheum Dis (2017) 76:e47. doi:10.1136/annrheumdis-2017-211446

46. Saito S, Suzuki K, Yoshimoto K, Kaneko Y, Matsumoto Y, Yamaoka K, et al. A new bioassay for measuring the strength of IL-6/STAT3 signal inhibition by tocilizumab in patients with rheumatoid arthritis. Arthritis Res Ther (2017) 19:231. doi:10.1186/s13075-017-1434-6

47. Chavele KM, Merry E, Ehrenstein MR. Cutting edge: circulating plasmablasts induce the differentiation of human T follicular helper cells via IL-6 production. J Immunol (2015) 194:2482-5. doi:10.4049/jimmunol.1401190

48. Aletaha D, Neogi T, Silman AJ, Funovits J, Felson DT, Bingham CO III, et al. 2010 rheumatoid arthritis classification criteria: an American College of Rheumatology/European League against rheumatism collaborative initiative. Arthritis Rheum (2010) 62:2569-81. doi:10.1002/art.27584

Conflict of Interest Statement: The authors declare that the research was conducted in the absence of any commercial or financial relationships that could be construed as a potential conflict of interest.

Copyright () 2018 Deng, Fan, Gao, Zeng, Guo, Wei, Chen, Chen, Gong, Feng, Xia, Xiang, Gong, Yuan, Shen, Shen, Lin, Jiang, He, Lu, Chen and Yu. This is an open-access article distributed under the terms of the Creative Commons Attribution License (CC BY). The use, distribution or reproduction in other forums is permitted, provided the original author(s) and the copyright owner are credited and that the original publication in this journal is cited, in accordance with accepted academic practice. No use, distribution or reproduction is permitted which does not comply with these terms. 